Pathologe 2010 · [Suppl 2] 31:215-220

DOI 10.1007/s00292-010-1344-8

Online publiziert: 22. August 2010

(c) Springer-Verlag 2010

\author{
K. Simon-Keller ${ }^{1}$ A. Paschen ${ }^{2}$ S. Eichmüller ${ }^{3}$ - S. Gattenlöhner ${ }^{4}$ - S. Barth ${ }^{5}$. \\ E. Koscielniak ${ }^{6} \cdot$ I. Leuschner ${ }^{7} \cdot$ P. Stöbel ${ }^{1} \cdot$ A. Hombach ${ }^{8} \cdot$ H. Abken ${ }^{8} \cdot$ A. Marx $^{1}$ \\ ${ }^{1}$ Pathologisches Institut, Universitätsmedizin Mannheim, \\ Universität Heidelberg, Mannheim \\ ${ }^{2}$ Klinik für Dermatologie, Universitätsklinikum Essen \\ ${ }^{3}$ Deutsches Krebsforschungszentrum, Heidelberg \\ ${ }^{4}$ Pathologisches Institut, Medizinische Universität Graz, Österreich \\ ${ }^{5}$ Molekularbiologie, Frauenhofer Institut für Molekularbiologie \\ und Angewandte Ökologie, Aachen \\ ${ }^{6}$ Olga Hospital, Stuttgart \\ ${ }^{7}$ Deutsches Kindertumorregister, Universitätsklinikum Schleswig-Holstein, \\ Campus Kiel \\ ${ }^{8}$ Tumorgenetik und Immunologie, Zentrum für Molekulare Medizin Köln
}

\title{
Adoptive T-Zell-Therapie des Rhabdomyosarkoms
}

morspezifisches „Target“ für eine adoptive Immuntherapie an.

Der erfolgreiche Einsatz von genetisch modifizierten T-Zellen als therapeutische Effektoren mit definierter Spezifität gegenüber einem Tumorantigen wurde bei zahlreichen Tumoren gezeigt, einschließlich Lymphomen [8], Melanomen [9] und Adenokarzinomen [10]. T-Zellen mit fAChR-spezifischem CAR (chimärer Antigenrezeptor) der 1. Generation zeigten eine geringe zytolytische Effektivität gegenüber RMS-Zellen in vitro und eine ungewöhnlich protrahierte Zytolyse [11].

In der vorliegenden Arbeit wurde nachgewiesen, dass RMS-Zellen kostimulatorische Liganden nur gering exprimieren, so dass wir einen CAR der 2. Generation mit kombinierter $\mathrm{CD}_{2} 8-\mathrm{CD}_{3} \zeta-\mathrm{Si}$ gnaldomäne generierten, um die T-ZellAktivierung zu verbessern.

\section{Ergebnisse}

\section{fAChR-Expression auf RMS-Zelllinien}

Um die Bindungsspezifität des „Singlechain-Antikörper-Fragmentes" zu testen, wurde ein scFv-Fc-Fragment ohne Transmembrandomäne generiert und in 293THEK-Zellen exprimiert. Dieser synthetische Antikörper wird von den 293T-Zel- len in den Kulturüberstand sezerniert und zur durchflusszytometrischen Detektion verwendet. Die Expression des fAChR wurde in 3 etablierten RMS-Zelllinien nachgewiesen (• Abb. 1). Die durchflusszytometrischen Profile gleichen denen nach Färbung mit kommerziellen Antikörpern (nicht gezeigt).

\section{RMS-Zellen exprimieren \\ keine CD54-, CD80-, CD86-kostimulatorischen Oberflächenmoleküle}

RMS scheinen diverse molekulare Mechanismen aufzuweisen, um einen "Angriff“ durch das Immunsystem zu umgehen. Unter anderem zeigen alle untersuchten RMS-Zelllinien eine verminderte Expression der Oberflächenmoleküle $\mathrm{CD}_{54}$ (ICAM1), CD8o und CD86 (• Abb. 2).

Neben den hier dargestellten Molekülen wurden weitere, immunologisch relevante Moleküle mit potenzieller Oberflächenexpression mittels qRT-PCR (quantitativer Real-time-Polymerase-Ketten-Reaktion) untersucht (nicht gezeigt). Verminderte Expression wurde $u$. a. bei CD7o, ICOS-L, TNFSF4 und TNFSF9 detektiert.

Diese Daten geben uns die Rationale, für die Aktivierung von T-Zellen gegen RMS-Zellen einen CAR zu generieren, 


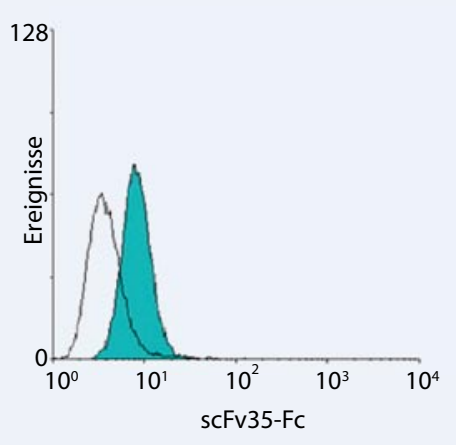

CRL2061

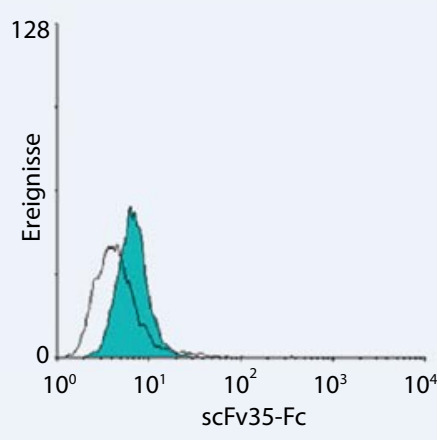

FIOH1

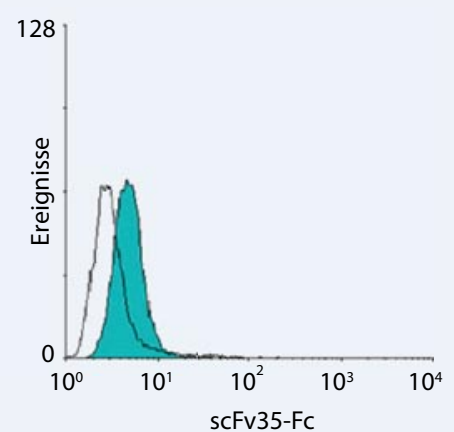

TE671
Abb. $1<$ Oberflächenexpression der $\gamma$-Untereinheit des Acetylcholinrezezeptors bei 3 RMS-Zelllinien: FACS-Analyse unter Verwendung des synthetische scFv-Fc-Antikörpers ( $g$ efüllte Histogramme), der die Bindestelle des in Abb. 3 gezeigten CAR darstellt. Die offenen Histogramme zeigen Färbungen mit einem Isotypantikörper
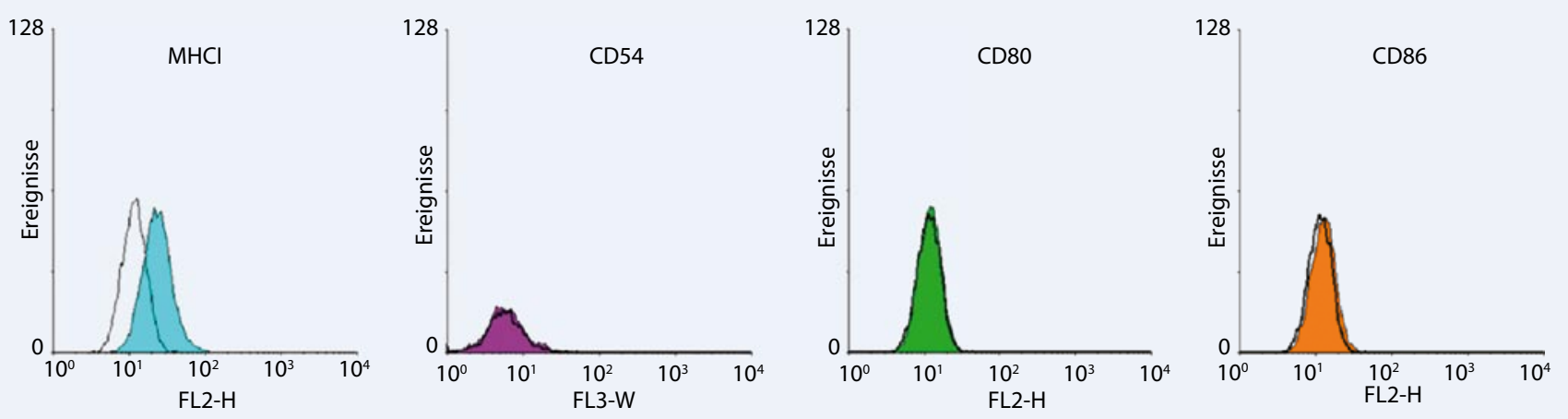

Abb. $2 \Delta$ Expression der Oberflächenmoleküle MHC-Klasse-I (MHCI), CD54, CD80 und CD86 auf RMS-Zellen. Als offene Histogramme dargestellt sind die Isotypkontrollen. Als Positivkontrollen dienten Melanom- und Lymphomzellen (nicht gezeigt)

der neben der $\mathrm{CD}_{3} \zeta$-Domäne die $\mathrm{CD}_{2} 8$ Signaldomäne trägt, um die vollständige T-Zell-Aktivierung zu gewährleisten.

\section{Erzeugung des chimären Antigenrezeptors mit der CD28-CD3ל-Signaldomäne}

Grundlage für die Bindungsdomäne zur Erkennung von RMS war ein Antikörper, der von einer Patientin stammte, deren Fötus/Kind an Arthrogryposis multiplex congenita litt, während die Patientin selbst klinisch unauffällig war [12]. Ursächlich für die Erkrankung des Fötus waren gegen die $\gamma$-Untereinheit des fAChR gerichtete Antikörper der Patientin $[13,14]$. Aus dem resezierten Thymus der Patientin wurde mittels „Phage Display" ein rekombinates Fab-Antikörper-Fragment mit Spezifität für die $\gamma$-Untereinheit des fAChR generiert. Aus diesem Fab-Fragment wurde wiederum ein "Single-chain-VH-VL-Fragment" mittels PCR kloniert, das als Ausgangsmaterial für Immunotoxine und CAR der T-Zellen $\operatorname{dient}(\mathrm{e})[7,11]$.

Ausgehend von dem retroviralen Vektor scFv35-SFG wurde das ,Single-chain-
Antikörper-Fragment" mit Spezifität für den fAChR in Vektoren umkloniert, die für $\mathrm{CAR}$ mit $\mathrm{CD}_{3} \zeta$ - oder $\mathrm{CD}_{2} 8-\mathrm{CD}_{3} \zeta$ Signaltransduktionsdomäne kodieren (- Abb. 3, unten).

Zur Modifikation der T-Zellen wurden die jeweiligen Vektoren transduziert und die CAR auf der Oberfläche der T-Lymphozyten exprimiert. Dabei wurden zunächst 293T-Zellen als Verpackungszelllinie mit den retroviralen Transduktionsvektoren und 2 Verpackungsplasmiden transfiziert, woraufhin die produzierten Viren zur Infektion der Lymphozyten verwendet wurden (• Abb. 3, oben). Der chimäre Rezeptor wurde mittels Durchflusszytometrie auf der Oberfläche der Lymphozyten detektiert. • Abb. 4 zeigt die Expression des CAR auf der Oberfläche von etwa $55 \%$ der Blutlymphozyten nach 3 Wochen In-vitro-Kultur.

\section{Zytotoxizität der CAR-modifizierten T-Zellen gegen RMS-Zellen in vitro}

T-Zellen mit fAChR-spezifischem CAR wurden mit RMS-Zellen in vitro kokultiviert und das Überleben der RMS-Zellen bestimmt (• Abb.5). Die verschie- denen RMS-Zelllinien wurden unterschiedlich stark lysiert. Dabei zeigten die T-Zellen mit einem $\mathrm{CD}_{28} 8-\mathrm{CD}_{3} \zeta$-Konstrukt die höchste lytische Aktivität. Verglichen wurden 3 alveoläre RMS-Zelllinien (CRL2061, $\mathrm{RH}_{3} \mathrm{O}$ und $\mathrm{RH}_{41}$ ) mit 3 embryonalen RMS-Zelllinien ( $\mathrm{FlOH} 1$, RD6 und TE671), deren fAChR-Expression ähnlich hoch war. Als Negativkontrolle dienten nichtmodifizierte periphere Blutlymphozyten (PBLs) sowie T-Zellen, die einen CAR mit Spezifität für CEA (karzinoembryonales Antigen) exprimieren. Als fAChR-negative Zelllinie dienten 293T-HEK-Zellen und eine CEA exprimierende Adenokarzinomzelllinie.

Dabei wurden unter den alveolären RMS-Linien die höchsten Lyseraten bei $\mathrm{RH}_{41}$-Zellen, unter den embryonalen Linien bei FlOH1-Zellen beobachtet. Mit abnehmender Effektorzellzahl („Effektor:Target-Ratio“) wurde eine verminderte Zelllyse und dadurch ein besseres Überleben der RMS-Zellen verzeichnet (• Abb. 4). Die beiden AChR-negativen Zelllinien $293 \mathrm{~T}$ und LS174 $\mathrm{T}$ zeigten nach Kokultivierung mit den entsprechenden T-Zellen eine kaum veränderte Überlebensrate, während die CEA-positive Zell- 
linie LS174T nach Kokultivierung mit TZellen mit dem Anti-CEA-CAR eine erwartete Zelllyse aufwies. Die Aktivierung der modifizierten T-Zellen durch den CAR wurde anhand der Interferon- (IFN-) $\gamma$-Sekretion bestätigt (nicht gezeigt).

\section{Diskussion}

Im Verlauf dieser Arbeit konnten wir zeigen, dass ein auf humanen T-Zellen rekombinant exprimierter CAR gegen den fAChR sein „Target“ spezifisch bindet. Im Gegensatz zu einer früheren Arbeit $[7,11]$ war es uns mit den modifizierten CAR möglich, bereits nach 48 Stunden eine Zelllyse nachzuweisen. Dennoch entspricht die beobachtete Lyse bei RMS-Zellen bei Weitem nicht derjenigen, die bei Lymphomen [15] oder Adenokarzinomen [10] durch T-Zellen mit entsprechenden CAR erreicht werden.

Ein entscheidender Faktor, der die Lyse von RMS-Zellen limitieren könnte, ist die im Vergleich zu CD2o auf B-Zell-Lymphomen oder zu CEA auf Adenokarzinomen des Kolons verminderte Expression des "Targets“ fAChR auf der Oberfläche von RMS-Zellen. Studien zur Relevanz der Dichte von „Target-Strukturen“ bei Adenokarzinomzellen zeigen, dass neben der Spezifität des chimären Immunorezeptors die „Target-Dichte“ wie auch die Bindungsaffinität für die Lyseraten der Tumorzellen von entscheidender Bedeutung ist [16]. Eine erhöhte Bindeaffinität für fAChR oder verbesserte Expression des CAR ist eine Möglichkeit, um die Effektivität der adoptiven Immuntherapie zu verbessern.

Andererseits könnte die fAChR-Expression auf den RMS-Zellen erhöht werden, wie es durch Chemotherapeutika induziert werden kann [11]. Erste Daten scheinen die Bedeutung dieses Ansatzpunktes für eine verbesserte adoptive Therapie von RMS zu bestätigen. Eine Kombination aus Chemotherapie und anschließender adoptiver Immuntherapie könnte künftig zur Eliminierung solcher RMS-Zellen beitragen, die auf pharmakologischem Weg allein bisher nicht eliminiert werden können.

Kostimulatorische Oberflächenmoleküle auf RMS-Zellen haben weiterhin Einfluss auf die T-Zell-Aktivierung. Zel-

Pathologe 2010 · [Suppl 2] 31:215-220 DOI 10.1007/s00292-010-1344-8

C) Springer-Verlag 2010

K. Simon-Keller · A. Paschen · S. Eichmüller · S. Gattenlöhner · S. Barth · E. Koscielniak · I. Leuschner · P. Stöbel · A. Hombach · H. Abken · A. Marx Adoptive T-Zell-Therapie des Rhabdomyosarkoms

\section{Zusammenfassung}

Zielsetzung. Um das Überleben bei fortgeschrittenen Rhabdomyosarkomen (RMS) zu verbessern, versuchen wir, Bedingungen für den adoptiven Transfer chimärer T-Zellen zu verbessern, die eine Spezifität für den fetalen Acetylcholinrezeptor, ein RMS-spezifisches Oberflächenmolekül, haben.

Methoden. Zur Optimierung der Rezeptorzytotoxizität wurde dem ursprünglich vorhandenen chimären Rezeptor, bestehend aus einem humanen Anti-fAChR-Antikörper, einer Fc-Hinge-Region und einer humanen CD3Z-Kette, eine CD28-Domäne zugefügt. Periphere Blutlymphozyten wurden mittels retroviraler Transduktion modifiziert und die Expression des chimären Rezeptors mittels Durchflusszytometrie verifiziert. Das zytotoxische Potenzial der modifizierten T-Zellen gegenüber RMS-Zellen wurde über MTTZytotoxizitätstests bestimmt. Die Expression kostimulatorischer Moleküle und antiapoptotischer Faktoren wurde durchflusszytometrisch bzw. mithilfe quantitativer PCR untersucht.

Ergebnisse. Die geringen Expressionslevel kostimulatorischer Moleküle auf RMS-Zel-

\section{Adoptive T-cell therapy of rhabdomyosarcoma}

\section{Abstract}

Aims. To improve survival of patients with advanced rhabdomyosarcomas (RMS), we aimed to adoptively transfer T-cells with redirected specificity for the fetal acetylcholine receptor (AChR), an RMS-specific cell surface antigen.

Methods. A "second generation" chimeric antigen receptor (CAR) with a combined $\mathrm{CD} 28-\mathrm{CD} 3$ З signaling domain was derived from our previously described chimeric antigen receptor composed of an extracellular human anti-fAChR antibody fragment, an Fc hinge region, and the intracellular T-cell receptor zeta chain. Lymphocytes from the peripheral blood were modified by retroviral transduction and monitored by FACS analysis. Cytotoxicity of modified T-cells towards RMS cells was recorded by MTT-based viability tests; expression of co-stimulatory molecules and anti-apoptotic genes was studied by FACS and qRT-PCR analysis.

Results. Co-stimulatory molecules were expressed in low levels on RMS cells giving the len führten zu der Überlegung, einen chimären Antigenrezeptor (CAR) mit einer CD28CD3そ-Signaldomäne zu generieren. Die erfolgreiche Modifikation der T-Zellen mit der „zweiten Generation" des AChR-spezifischen CAR zeigte trotz guter Expressionsraten geringe Lyseraten gegenüber RMS, im Vergleich zu CD20-positiven Lymphom- oder CEA-exprimierenden Adenokarzinomzelllinien mit CD20- bzw. CEA-spezifischem CAR.

Schlussfolgerung. Die verminderten Lyseraten der RMS-Zellen durch einen AChR-spezifischen CAR lassen eine Resistenz der RMSZellen gegenüber der T-Zell-Antwort vermuten. Die Inhibierung antiapoptotischer Stoffwechselwege könnte die Sensitivität dieser Zellen gegenüber konventioneller und auch gegenüber T-Zell-basierten Therapien verbessern.

\section{Schlüsselwörter}

Rhabdomyosarkom · Fetaler Acetylcholinrezeptor · AChR- $\gamma \cdot$ T-Zellen · Chimärer Antigenrezeptor rationale to generate a $\mathrm{CD} 28-\mathrm{CD} 3$ / signalling CAR (chimeric antigen receptor) for redirecting T-cells. T-cells were successfully engineered with the "second generation" AChRspecific chimeric antigen receptor. Despite of high CAR expression engineered T-cells showed low killing efficiency towards RMS compared to redirected killing of CD20+ lymphoma or CEA-expressing adenocarcinoma cell lines when redirected by CD20- and/or CEA-specific CAR.

Conclusions. Data suggest that RMS cells exhibit resistance to a T-cell attack redirected by a fAChR-specific CAR. Inhibition of anti-apoptotic pathways in those cells may improve sensitivity to conventional as well as T-cellbased therapeutics.

\section{Keywords}

Rhabdomyosarcoma - Acetylcholine receptor, fetal · AChR- $\gamma \cdot$ T-cells · Antigen receptor, chimeric 


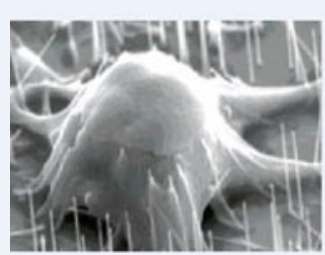

293T/GP2-293

Verpackungszelllinie
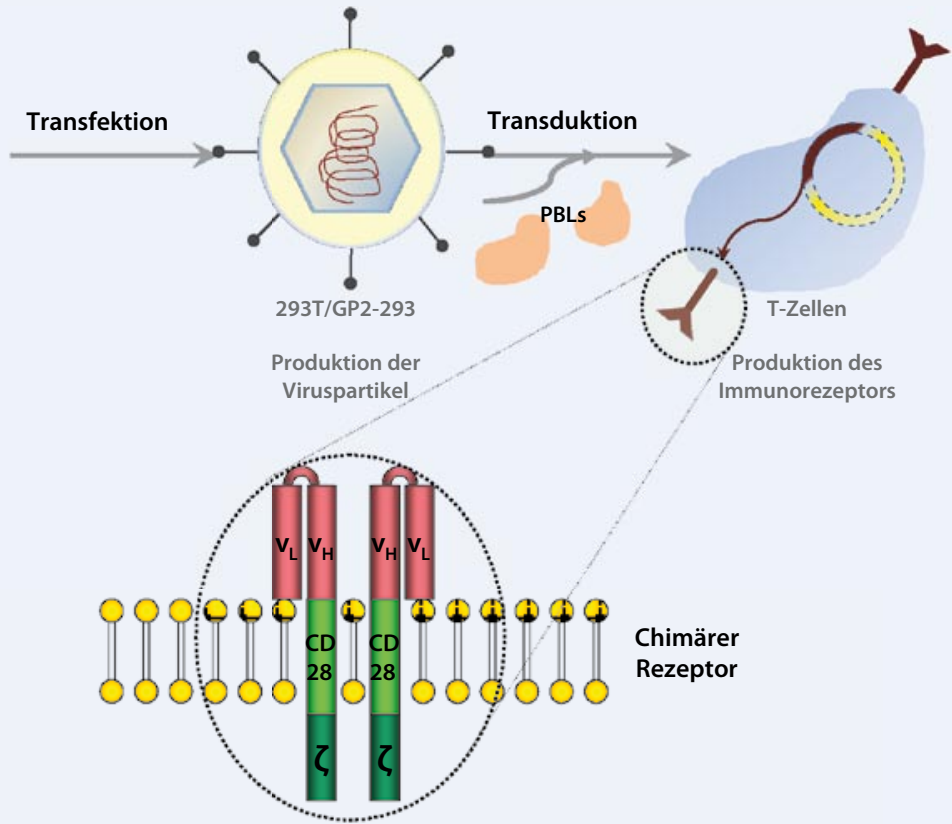

scFv35:

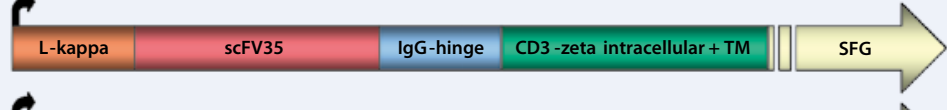

KSK1:
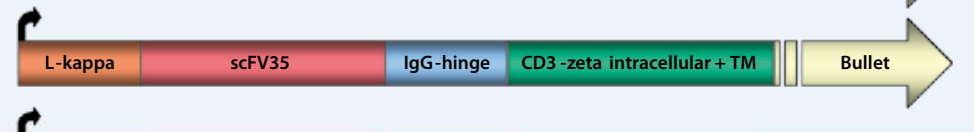

c
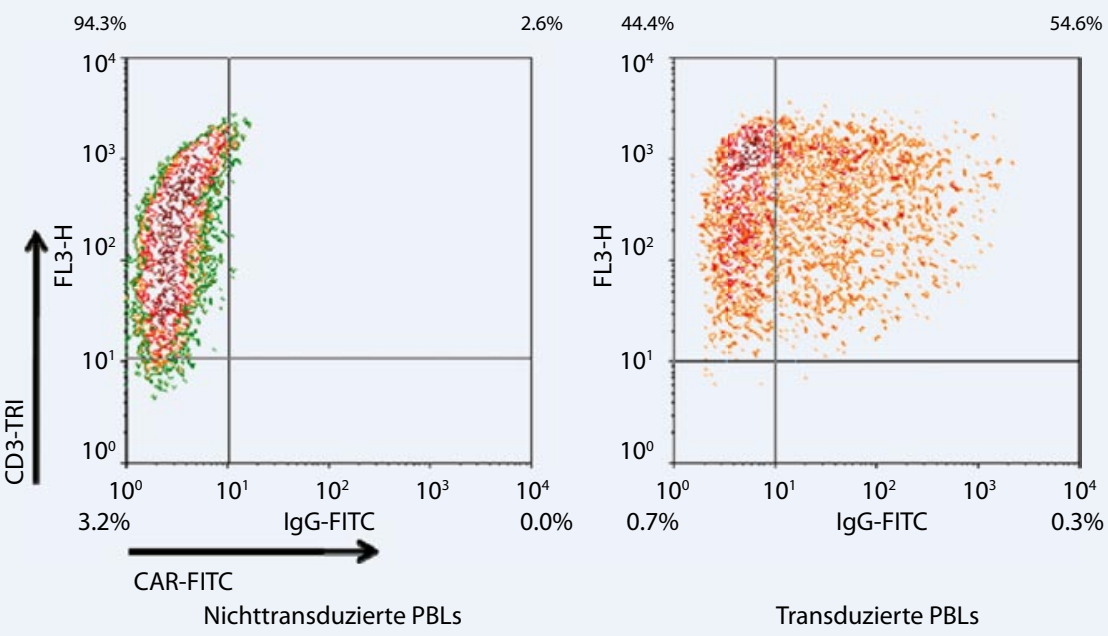

Abb. $4 \Delta$ Durchflusszytometrische Analyse der CAR-Expression auf der Oberfläche der T-Lymphozyten. Zur Detektion des CAR diente ein „Anti-human-IgG-FITC-markierter" Antikörper, der die extrazelluläre IgG-Fc-Domäne des CAR bindet. Zur Detektion der T-Zellen diente ein anti-humaner CD3-TRIAntikörper (PBLs periphere Blutlymphozyten)
Abb. $3<$ Oben: Prinzip der Modifikation peripherer Blutlymphozyten mit antiAChR-spezifischen CAR auf der Basis von retroviralen Vektoren. Unten: modularer Aufbau der CAR (TM Transmembrandomäne, PBLs periphere Blutlymphozyten)

len von RMS-Zelllinien zeigen ähnlich wie Myoblasten [17] eine sehr geringe bis fehlende Expression kostimulatorischer Rezeptoren wie ICAM1 [18], CD8o und CD86. Diese Moleküle spielen eine wesentliche Rolle bei der Interaktion von antigenpräsentierenden Zellen, immunologischen Effektorzellen und ihren Zielzellen. Durch die Verwendung eines CAR der 2. Generation, d. h. mit CD28-kostimulatorischer Signaldomäne zusätzlich zu $\mathrm{CD}_{3} \zeta$, haben wir die Möglichkeit geschaffen, eine vollständige T-Zell-Aktivierung auch bei fehlender CD80- und CD86-Expression der Zielzellen zu erreichen.

Zusammengefasst schließen wir aus unseren Beobachtungen, dass zwar die TZell-Aktivierung spezifisch und vollständig erfolgt, die Lyse der RMS-Zellen jedoch gering ist. Dieses deutet auf einen intrinsischen Mechanismus hin, der durch eine Apoptoseresistenz vermittelt sein kann. Andere Tumorzellen, wie z. B. Melanom- und Hodgkin-Lymphom-Zellen, 
weisen ebenfalls eine Apoptoseresistenz bei einer T-Zell-vermittelten Lyse auf, die jedoch durch pharmakologisches Targeting des Apoptosesignalweges verbessert werden konnte [19].

\section{Material und Methoden}

\section{Zelllinien und Antikörper}

Ein Teil der verwendeten Zelllinien wurden von Frau Prof. Ewa Koscielniak (Olgahospital, Stuttgart; Ax-OH1 und Fl$\mathrm{OH}_{1}$ ) und Frau PD Dr. Annette Paschen (Deutsches Krebsforschungszentrum/DKFZ, Heidelberg; RD6 und TE671, 293T) zur Verfügung gestellt. Die Zelllinien $\mathrm{RH}_{1}, \mathrm{RH}_{3} \mathrm{O}$ und $\mathrm{RH}_{41}$ wurden von der Deutschen Sammlung für Mikroorganismen und Zellkultur (DSMZ, Braunschweig) bezogen. Die Kultivierung erfolgte nach Vorgaben der DSMZ.

Die beiden retroviralen Vektoren zur Erzeugung des CAR und der Vektor zur Expression des löslichen humanen „Single-chain-Antikörper-Fragmentes" scFvFc gegen fAChR wurden von Herrn Prof. Hinrich Abken (Zentrum für Molekulare Medizin, Uniklinik Köln) bereitgestellt. Die verwendete fAChR-spezifische Bindestruktur entspricht dem scFv35/VLVH [11].

Humane T-Zellen wurden aus dem Blut gesunder Spender gereinigt und einmalig mit $0,5 \mu \mathrm{g} / \mathrm{ml}$ OKT-3-Antikörper (BioLegend) und $0,5 \mu \mathrm{g} / \mathrm{ml}$ Anti-CD28Antikörper (BD) aktiviert und in Gegenwart von $400 \mathrm{U} / \mathrm{ml}$ Interleukin-2 (Novartis) in Kulturmedium inkubiert.

\section{Retrovirale Transduktion}

Die Transduktion der retroviralen Vektoren in humane Lymphozyten erfolgte nach etablierten Verfahren. Die Expression des CAR auf T-Zellen wurde nach Färbung mit „Anti-human-IgG ${ }_{1}$-Antikörper“ und "Anti-human-CD3-Antikörper" mittels Durchflusszytometrie bestimmt.

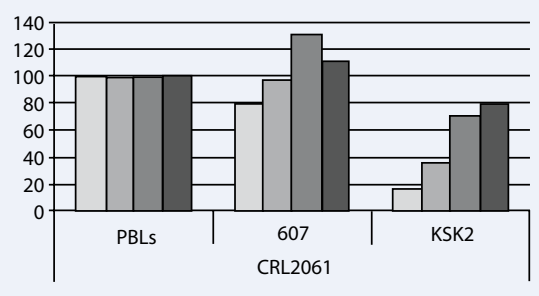

$\square 5,00 E+04 \square 2,50 E+04 \square 1,25 E+04 \square 6,25 E+03$

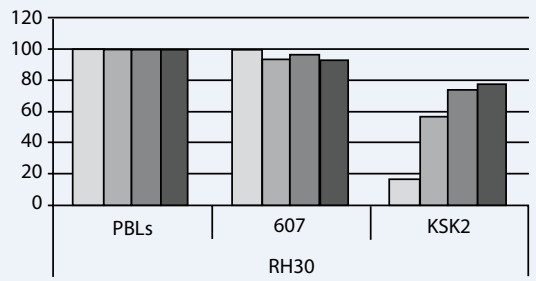

$\square$ 5,00E+04 $\square 2,50 \mathrm{E}+04 \square 1,25 \mathrm{E}+04 \square 6,25 \mathrm{E}+03$

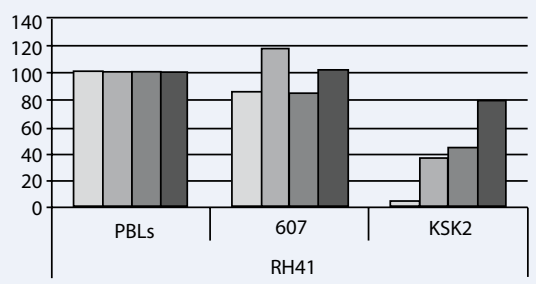

$\square$ 5,00E+04 $\square$ 2,50E+04 $\square$ 1,25E+04 $\square$ 6,25E+03

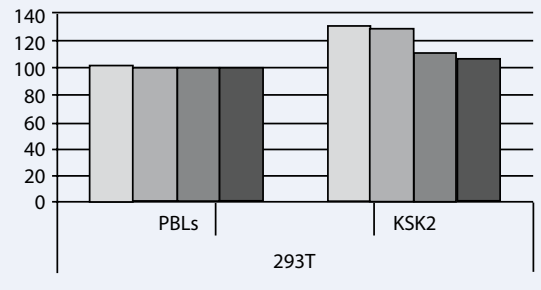

$\square 5^{*} 104 \square 2,5^{*} 104 \square 1,25 * 104 \square 0,625^{*} 104$

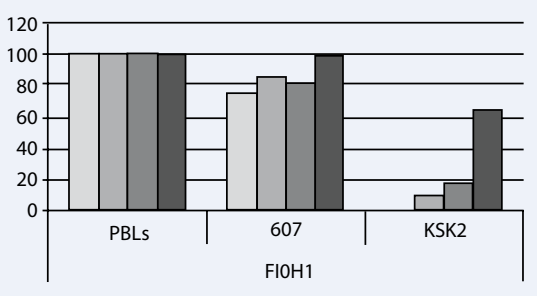

$\square 5,00 \mathrm{E}+04 \square 2,50 \mathrm{E}+04 \square 1,25 \mathrm{E}+04 \quad \square 6,25 \mathrm{E}+03$

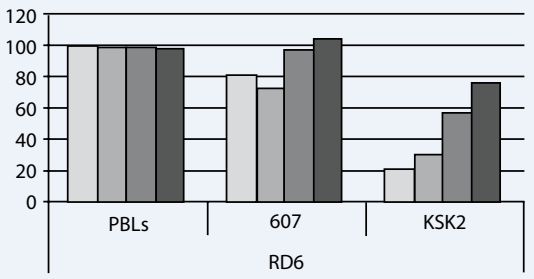

$\square$ 5,00E+04 $\square$ 2,50E+04 $\square$ 1,25E+04 $\square$ 6,25E+03

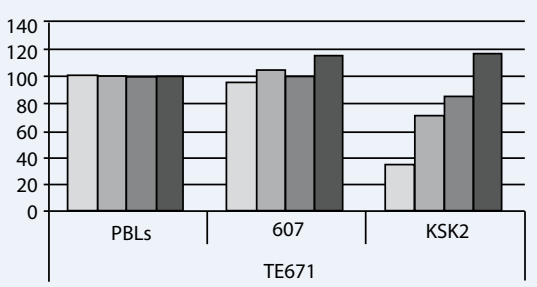

$\square$ 5,00E+04 $\square$ 2,50E+04 $\square$ 1,25E+04 $\square$ 6,25E+03

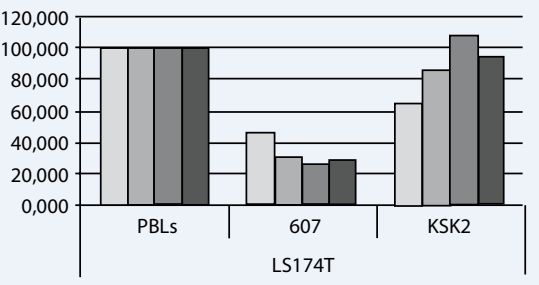

$\square$ 5,00E+04 $\square$ 2,50E+04 $\square$ 1,25E+04 $\square$ 6,25E+03

Abb. 5 - Überleben der RMS-Zellen nach Kokultivierung mit CAR-modifizierten T-Zellen. Dargestellt sind die Überlebensraten in Prozent bezogen auf die Kokultivierung von RMS-Zellen mit nichttransduzierten PBLs. Die Graphen stellen verschiedene Effektorzellkonzentrationen (5-mal 104 bis 0,625-mal 104 Zellen/"well") bei konstanter Target-Zellkonzentration (1-mal 104 RMS-Zellen/“well") dar

\section{Zytotoxizitätstests}

Die transduzierten T-Zellen $\left(5-\right.$ mal $_{10} \mathrm{O}^{4}$ bis 0,625-mal 10 $0^{4}$ Zellen/“well“) wurden für 72 Stunden in 96-well-Rundboden-Platten mit den RMS-Zellen (1-mal 104 Zellen/ "well" bei FlOH1, RD6 und TE671; 2,5-mal $10^{4}$ Zellen/“well" bei CRL2061, $\mathrm{RH}_{30}$ und RH41) kokultiviert. Die Kulturüberstände wurden hinsichtlich Konzentration von IFN- $\gamma$ mittels ELISA (Mabtech, human IFNgamma Elisa-ALP) untersucht. Die Vitalität der Zellen wurde durch Zugabe von MTT (Methylthiazolyldiphenyl-tetrazolium bromide, Sigma Aldrich, $5 \mathrm{mg} / \mathrm{ml}$ ) bestimmt. Die Reduktion von MTT zu Formazan durch vitale Tumorzellen wurde mittels „Mikroplatten Reader" (TECAN, Infinite 200) bei einer Wellenlänge von $560 \mathrm{~nm}$ und einer Referenzwellenlänge von $670 \mathrm{~nm}$ bestimmt. Die nichtspezifische Aktivität der Effektorzellen wurde durch Kultivierung von Effektorzellen ohne Tumorzellen bestimmt (s. Formel).

OD (Kokultivierung von Tumor- und Effektorzellen - korrespondierende Anzahl von Effektorzellen) $\times 100$

Vitale Tumorzellen $[\%]=\frac{\text { OD (Tumorzellen ohne Effektorzellen - Medium) }}{\text { O }}$ 


\section{Korrespondenzadresse}

\section{K. Simon-Keller}

Pathologisches Institut, Universitätsmedizin Mannheim, Universität Heidelberg Theodor-Kutzer-Ufer 1-3, 68167 Mannheim katja.simon-keller@medma.uni-heidelberg.de

Interessenkonflikt. Die korrespondierende Autorin gibt an, dass kein Interessenkonflikt besteht.

\section{Literatur}

1. Miller RW, Y.J.J, Novakovic B (1995) Childhood cancer. Cancer 75(S1):395-405

2. Crist W, Gehan EA, Ragab AH et al (1995) The third intergroup rhabdomyosarcoma study. J Clin Oncol 13:610-630

3. Carli M et al (1988) Tumor response and toxicity after single high-dose versus standard five-day divided-dose dactinomycin in childhood rhabdomyosarcoma. J Clin Oncol 6(4):654-658

4. Gatta G et al (2005) Childhood cancer survival trends in europe: a EUROCARE working group study. J Clin Oncol 23(16):3742-3751

5. Dantonello TM et al (2009) Cooperative trial CWS91 for localized soft tissue sarcoma in children, adolescents, and young adults. J Clin Oncol 27(9):1446-1455

6. David AR, Anderson JR, Arndt CA et al (2010) Comparison of outcomes based on treatment algorithms for rhabdomyosarcoma (RMS) of the bladder/ Prostate (BP): combined results from the Children's Oncology Group (COG), German Cooperative Soft Tissue Sarcoma Study (CWS), Italian Cooperative Group (ICG), and International Society of Pediatric Oncology (SIOP) Malignant Mesenchymal Tumors (MMT) committee. Int J Cancer [Epub ahead of print May 11]

7. Gattenloehner S, Vincent A, Leuschner I et al (1988) The fetal form of the acetylcholine receptor distinguishes rhabdomyosarcomas from other childhood tumors. Am J Pathol 152(2):437-444

8. Landmeier S, Altvater B, Pscherer S et al (2010) Cytotoxic T cells transduced with chimeric anti-CD19 receptors prevent engraftment of primary lymphoblastic leukemia in vivo. Leukemia 24(5):1080 1084

9. Dudley ME, Yang JC, Sherry R et al (2008) Adoptive cell therapy for patients with metastatic melanoma: evaluation of intensive myeloablative chemoradiation preparative regimens. J Clin Oncol 26(32):5233-5239

10. Hombach A, Schlimper C, Sievers E et al (2006) A recombinant anti-carcinoembryonic antigen immunoreceptor with combined CD33-CD28 signalling targets $T$ cells from colorectal cancer patients against their tumour cells. Gut 55:1156-1164

11. Gattenlöhner S, Marx A, Markfort B et al (2006) Rhabdomyosarcoma lysis by $T$ cells expressing a human autoantibody-based chimeric receptor targeting the fetal acetylcholine receptor. Cancer Res 66:24-28

12. Matthews I, Sims G, Lewidge S et al (2002) Antibodies to acetylcholine receptor in parous women with myasthenia: evidence for immunization by fetal antigen. Lab Invest 82(10):1407-1417

13. Matthews I, Farrar J, McLachlan S et al (1998) Production of fab fragments against the human acetylcholine receptor from myasthenia gravis thymus lambda and kappa phage libraries. Ann NY Acad Sci 841:418-421
14. Vincent A, Palace J, Hilton-Jones D (2001) Myasthenia gravis. Lancet 357(9274):2122-2128

15. Torsten D, Lorenczewski G, Brandl C et al (2002) Extremely potent, rapid and costimulation-independent cytotoxic T-cell response against lymphoma cells catalyzed by a single-chain bispecific antibody. Int J Cancer 100(6):690-697

16. Chmielewski M, Hornbach A, Heuser C et al (2004) $T$ cell activation by antibody-like immunoreceptors: increase in affinity of the single-chain fragment domain above threshold does not increase $T$ cell activation against antigen-positive target cells but decreases selectivity. J Immunol 173(12):7647-7653

17. Wiendl H, Hohlfeld R, Kieseier BC (2005) Musclederived positive and negative regulators of the immune response. Curr Opin Rheumatol 17(6):714719

18. Pui CH et al (1993) Serum intercellular adhesion molecule- 1 in childhood malignancy. Blood 82(3):895-898

19. Kashkar H, Deggerich A, Seeger J-M et al (2007) NF-kB-independent down-regulation of XIAP by bortezomib sensitizes HL B cells against cytotoxic drugs. Blood 109(9):3982-3988 